\title{
Single-Step Rapid Diagnosis of Dopamine and Serotonin Metabolism Disorders
}

Aurélien Lo, ${ }^{\dagger}$ Pierre Guibal, $^{\dagger}$ Diane Doummar, ${ }^{\ddagger}$ Diana Rodriguez, ${ }^{\ddagger}$ Jean-Yves Hautem, ${ }^{\ddagger}$ Rémy Couderc, ${ }^{\ddagger}$ Thierry Billette De Villemeur, Emmanuel Roze, ${ }^{\S, \|}$ Pierre Chaminade, ${ }^{\dagger}$ and Fathi Moussa*, ${ }^{\ddagger}, \neq$

${ }^{\dagger}$ Letiam, Lip $(\text { Sys })^{2}$, EA7357, IUT d’Orsay, Université Paris Sud, Plateau de Moulon, 91400 Orsay, France

${ }^{\ddagger}$ Services de Neuropédiatrie et de Biochimie, Groupe Hospitalier Trousseau-Laroche-Guyon, 26 avenue du Dr Arnold Netter, 75012 Paris, France

${ }^{\S}$ Department of Neurology, Pitié-Salpêtrière Hospital, AP-HP, 75013 Paris, France

"UMR S 952, INSERM, Paris 6 University, 75005 Paris, France

\section{Supporting Information}

ABSTRACT: Early diagnosis of dopamine and serotonin metabolic defects is of importance notably because of the availability of therapeutic strategies able to prevent the associated progressive brain dysfunction. The diagnosis of these diseases relies on the determination of monoamine metabolites and pterins in cerebrospinal fluid (CSF). Current methods involve at least two high-performance liquid chromatography runs of CSF analysis. The first one is devoted to the quantification of dopamine and serotonin metabolites and the second one to the quantification of pterins. Here, we describe a single-step method to measure monoamine neurotransmitter metabolites and pterins of interest in less

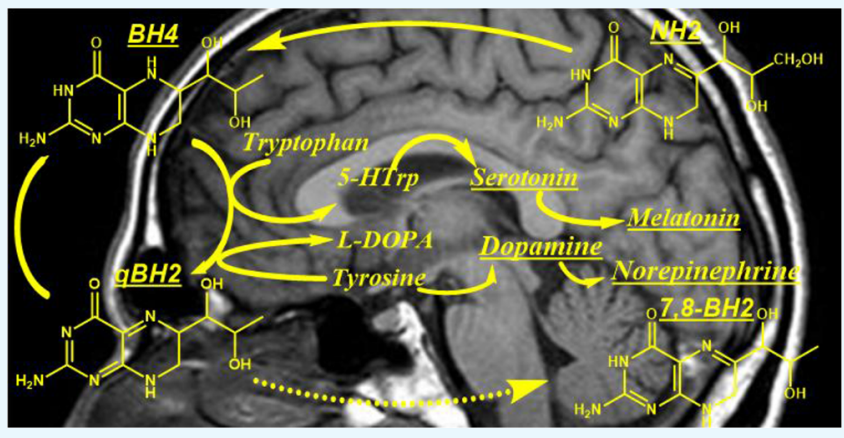
than $10 \mathrm{~min}$ by ultrahigh-performance liquid chromatography coupled to sequential coulometric oxidation and fluorescence detections. All target compounds were quantified in CSF with a small volume $(50 \mu \mathrm{L})$ and a single filtration step for sample preparation and analysis. After validation, the proposed method was applied to the determination of age-related reference ranges in the CSF of target compounds from a series of 1372 samples collected in France from 2008 to 2014. In the same period, the results obtained for 19 CSF samples from patients with known neurotransmitter disorders and 115 CSF samples with known immune system activation confirmed the expected pattern of changes in monoamine metabolites and pterins.

\section{INTRODUCTION}

Since the description of the Segawa syndrome, also known as dihydroxy-phenyl-alanine (DOPA)-responsive dystonia, several neurotransmitter-inherited metabolic disorders including biosynthesis, breakdown, or transport defects of dopamine and serotonin have been reported. ${ }^{1-3}$ Inherited defects leading to these metabolic diseases have been described or predicted at the level of each enzyme involved in the metabolism of dopamine and serotonin (Figure 1). ${ }^{1-3}$

Tetrahydrobiopterin (BH4), the cofactor of aromatic amino acid hydroxylases, plays a pivotal role in the synthesis of dopamine and serotonin (Figure 1). ${ }^{4-6}$ Hence, defects in the biosynthesis or regeneration of $\mathrm{BH} 4$ result in several dopamine and serotonin metabolic disorders. Each of these conditions exhibits a characteristic cerebrospinal fluid (CSF) profile of dopamine and serotonin precursors and metabolites (Table 1). ${ }^{4-6}$

As $\mathrm{BH} 4$ is also the cofactor of phenylalanine hydroxylase (Figure 1), BH4 deficiencies can be detected at the time of newborn screening except for autosomal dominant GTPCH deficiencies, certain cases of heterozygote GTPCH deficiencies, and autosomal recessive SR deficiencies, which do not lead to hyperphenylalaninemia (Table 1$).^{4-6}$

Early diagnosis of dopamine and serotonin metabolic disorders is of importance notably because some of these diseases can be well-treated. ${ }^{7-9}$ In addition to the determination of CSF neurotransmitter metabolites, the etiological diagnosis of these inherited disorders also requires the determination of pterins in CSF, notably dihydroneopterin (NH2), the precursor of $\mathrm{BH} 4$, and 7,8-dihyrobiopterin ( $\mathrm{BH} 2$ ), the stable oxidized form of the latter (Figure 1, Table 1). ${ }^{1-6}$

To sum it up, the differential diagnosis of dopamine and serotonin metabolic disorders requires the determination of homovanillic acid (HVA), 5-hydroxyindoleacetic acid (5-HIAA), 3-ortho-methyl-di-hydroxyphenylalanine (3-OMD), 5-hydroxytryptophan (5-HTrp), NH2, and $\mathrm{BH} 2$ in the CSF (Table 1). To this purpose, current methods require at least two chromatographic steps of CSF analysis. The first one is devoted to the

Received: July 17, 2017

Accepted: August 23, 2017

Published: September 19, 2017 


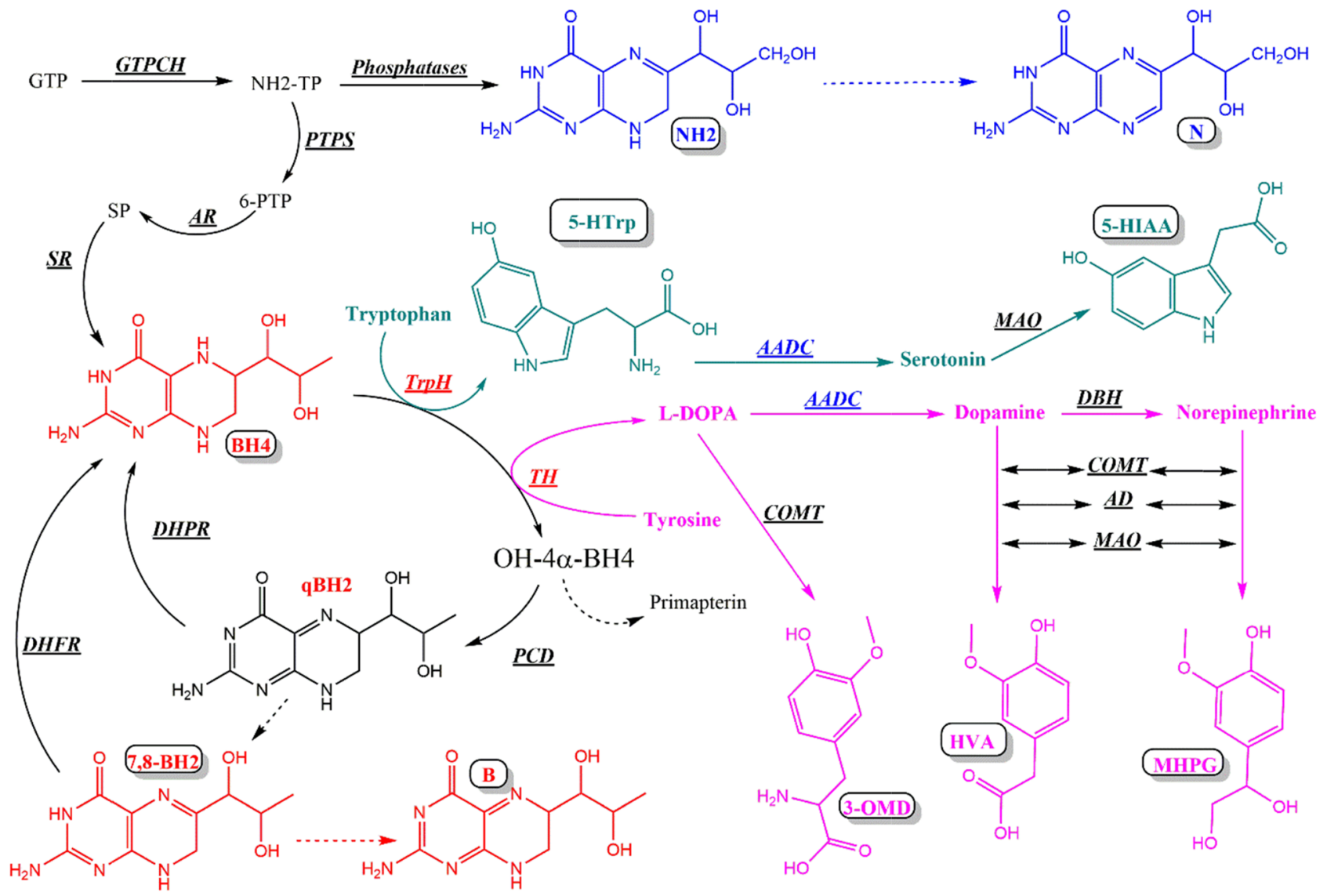

Figure 1. Tetrahydrobiopterin (BH4) and monoamine neurotransmitter metabolism. GTP (guanosine triphosphate), GTPCH (guanosine triphosphate cyclohydrolase), NH2-TP (dihydroneopterin triphosphate), NH2 (dihydroneopterin), N (neopterin), PTPS (6-pyruvoyltetrahydropterin synthase), AR (aldose reductase), SR (sepiapterin reductase), BH4 (tetrahydrobiopterin), PCD (pterin-4 $\alpha$-carbinolamine dehydratase), qBH2 (quinonoid dihydrobiopterin), DHPR (dihydropteridin reductase), 7,8-BH2 (dihydrobiopterin), DHFR (dihydrofolate reductase), B (biopterin), TrpH (tryptophan hydroxylase), 5-HTrp (5-hydroxytryptophan), AADC (aromatic amino acid decarboxylase, cofactor: pyridoxal phosphate), MAO (monoamine oxidase), 5-HIAA (5-hydroxyindoleacetic acid), TH (tyrosine hydroxylase), L-DOPA (Ldihydroxyphenylalanine), DBH (dopamine $\beta$-hydroxylase), COMT (catechol-O-methyltransferase), AD (aldehyde dehydrogenase), 3-OMD (3ortho-methyl DOPA), HVA (homovanillic acid), and MHPG (3-methoxy-4-hydroxyphenylglycol) (dashed arrows: nonenzymatic).

Table 1. Changes in CSF Neurotransmitter Metabolites and Pterins Concentrations in Disorders of Dopamine and Serotonin Metabolism (According to Refs 4-6)

\begin{tabular}{|c|c|c|c|c|c|c|c|c|c|c|}
\hline \multirow[b]{2}{*}{ enzymatic defect } & \multirow[b]{2}{*}{$\mathrm{Phe}^{a}$} & \multicolumn{3}{|c|}{ pterins } & \multicolumn{6}{|c|}{ neurotransmitter metabolites } \\
\hline & & $\mathrm{BH} 4$ & $\mathrm{BH} 2$ & $\mathrm{NH} 2$ & HVA & HIAA & HVA/HIAA & 3-OMD & 5-HTrp & MHPG \\
\hline GTPCH AR & $\uparrow$ & $\downarrow$ & $\mathrm{N}$ & $\downarrow$ & $\downarrow$ & $\downarrow$ & $\mathrm{N}$ & $\mathrm{N}$ & $\mathrm{N}$ & $\downarrow$ \\
\hline PTPS & $\uparrow$ & $\downarrow$ & $\mathrm{N}$ & $\uparrow$ & $\downarrow$ & $\downarrow$ & $\mathrm{N}$ & $\mathrm{N}$ & $\mathrm{N}$ & $\downarrow$ \\
\hline PCD & $\uparrow$ & $\downarrow$ & $\mathrm{N}$ & $\mathrm{N}$ & $\mathrm{N}$ & $\mathrm{N}$ & $\mathrm{N}$ & $\mathrm{N}$ & $\mathrm{N}$ & $\mathrm{N}$ \\
\hline DHPR & $\uparrow$ & $\downarrow$ & $\uparrow$ & $\mathrm{N}$ & $\downarrow$ & $\downarrow$ & $\mathrm{N}$ & $\mathrm{N}$ & $\mathrm{N}$ & $\downarrow$ \\
\hline GTPCH AD & $\mathrm{N}$ & $\downarrow$ & $\mathrm{N}$ & $\downarrow$ & $\downarrow$ & $\mathrm{N}$ or $\downarrow$ & $\downarrow$ & $\mathrm{N}$ & $\mathrm{N}$ & $\downarrow$ \\
\hline SR & $\mathrm{N}$ & $\downarrow$ & $\uparrow$ & $\mathrm{N}$ & $\downarrow$ & $\downarrow$ & $\mathrm{N}$ & $\mathrm{N}$ & $\mathrm{N}$ & $\downarrow$ \\
\hline $\mathrm{TH}$ & $\mathrm{N}$ & $\mathrm{N}$ & $\mathrm{N}$ & $\mathrm{N}$ & $\downarrow$ & $\mathrm{N}$ & $\downarrow$ & $\mathrm{N}$ & $\mathrm{N}$ & $\downarrow$ \\
\hline $\mathrm{AADC}$ & $\mathrm{N}$ & $\mathrm{N}$ & $\mathrm{N}$ & $\mathrm{N}$ & $\downarrow$ & $\downarrow$ & $\mathrm{N}$ & $\uparrow$ & $\uparrow$ & $\downarrow$ \\
\hline DTDS & $\mathrm{N}$ & $\mathrm{N}$ & $\mathrm{N}$ & $\mathrm{N}$ & $\uparrow$ & $\mathrm{N}$ & $\uparrow$ & $\mathrm{N}$ & $\mathrm{N}$ & $\mathrm{N}$ \\
\hline
\end{tabular}

${ }^{a}$ Phe (phenylalanine as determined in plasma), N (normal), GTPCH AR (GTP-cyclohydrolase deficiency autosomal recessive), PTPS (6-pyruvoyltetrahydropterin synthase deficiency), PCD (pterin- $4 \alpha$-carbinolamine dehydratase deficiency), DHPR (dihydropteridine reductase deficiency), GTPCH AD (GTP-cyclohydrolase deficiency autosomal dominant also known as Segawa syndrome or DOPA-responsive dystonia), SR (sepiapterin reductase deficiency), TH (tyrosine hydroxylase deficiency), AADC (aromatic L-amino acid decarboxylase deficiency), and DTDS (dopamine transporter deficiency syndrome).

quantification of dopamine and serotonin metabolites and the second one to the quantitation of pterins. ${ }^{10-19}$

As dopamine and serotonin metabolites are oxidizable compounds, high-performance liquid chromatography coupled to electrochemical detection (HPLC-ECD) is commonly used to quantify them in the CSF. ${ }^{10-17}$ More recently, some liquid chromatography-mass spectrometry (LC-MS) methods have also been implemented. ${ }^{19,20}$ On the other hand, the CSF pterin 

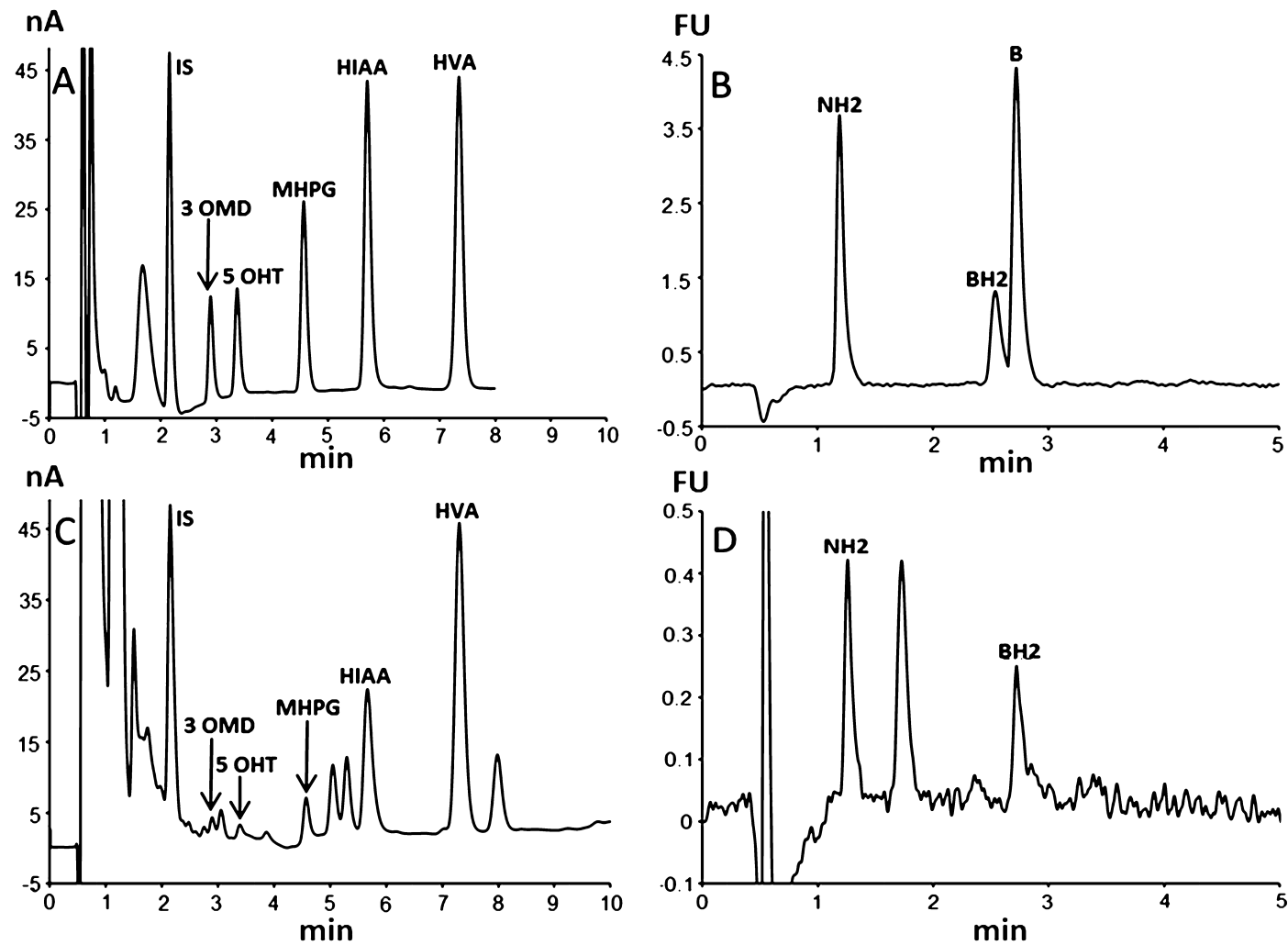

Figure 2. Chromatographic profiles obtained by the proposed method of (A,B) a standard mixture with (A) ECD followed by (B) sequential fluorescence detection and (C,D) a CSF sample of a patient from the control group with (C) ECD and (D) sequential fluorescence detection. Chromatographic conditions: stationary phase $=$ Atlantis $\mathrm{T} 3(4.6 \times 150 \mathrm{~mm}$ and $3 \mu \mathrm{m})$ column; mobile phase: $\mathrm{pH} 5.2,0.05 \mathrm{M}$ citrate buffer, and methanol $(97 / 3, \mathrm{v} / \mathrm{v})$. Flow rate was set at $0.5 \mathrm{~mL} / \mathrm{min}$ at $30^{\circ} \mathrm{C}$. The metabolites present in the effluent are measured at the second electrode with a potential set at $400 \mathrm{mV}$, whereas the fluorescence of pterins is sequentially measured at $450 \mathrm{~nm}$ after column electrooxidation at the same potential and excitation at $350 \mathrm{~nm}$ (FU: arbitrary fluorescence units).

concentrations are in the low nM range at basal levels. Thus, their direct quantification does require an HPLC method coupled to fluorescence or MS detections. ${ }^{1,10,11,17,19}$

Here, we describe a single-step ultrahigh-performance liquid chromatography (UHPLC) method for the rapid diagnosis of dopamine and serotonin metabolic disorders. Neurotransmitter metabolites as well as pterins of interest are determined in a single step in less than $10 \mathrm{~min}$ by UHPLC coupled to sequential electrochemical and fluorescence detections.

After validation, the proposed method requiring only a single filtration step for sample preparation and analysis was applied to the analysis of a series of 1650 CSF samples including several cases of known neurotransmitter disorders and known immune system activation.

\section{RESULTS AND DISCUSSION}

Method Development. Preliminary HPLC separations were achieved using the same polar-embedded C18 column $(4.6 \times 150 \mathrm{~mm}, 3 \mu \mathrm{m}$, and Atlantis T3) previously used for the separation of neurotransmitter metabolites ${ }^{17}$ and pterins. ${ }^{18}$ In contrast to classical Si-C18 columns, this stationary phase allows the separation of polar compounds including both neurotransmitter metabolites and pterins without using an ion-pairing reagent. $^{18}$

Systematic investigation of the effects of $\mathrm{pH}$, mobile phase composition, and temperature allowed us to obtain a separation with high efficiency and high resolution of all target compounds in less than $50 \mathrm{~min}$ (Figure S1A). High resolution is mandatory for the specific ECD and quantification of neurotransmitter metabolites because real CSF samples exhibit several unknown additional peaks (Figure S1C). The electrochemical detector also served as a postcolumn coulometric oxidation reactor for the oxidation of reduced pterins prior to sequential fluorescence detection (Figure S1B,D). Hence, this method allows the simultaneous separation of both neurotransmitter metabolites and pterins in a final run time of $50 \mathrm{~min}$.

To speed up the separation, we converted the HPLC method to UHPLC. For this purpose, we used an ACQUITY UPLC HSS T3 column $(2.1 \times 100 \mathrm{~mm}$ and $1.8 \mu \mathrm{m})$ with the same mobile phase as for the HPLC separation, delivered at a flow rate of 0.4 $\mathrm{mL} / \mathrm{min}$ at $30^{\circ} \mathrm{C}$. This conversion resulted in the separation of the whole of the analytes in less than $8 \mathrm{~min}$ (Figure 2) with a final run time of $10 \mathrm{~min}$ for real samples, thus allowing the complete elution of possible unknown peaks eluting after the HVA peak (Figure 2C).

The best separation in terms of efficiency and resolution is obtained with a mobile phase consisting of a mixture of $\mathrm{pH} 5.2$ and $0.05 \mathrm{M}$ citrate buffer and methanol $(97 / 3, \mathrm{v} / \mathrm{v})$. The $\mathrm{pH}$ of the mobile phase should not fall below 5.2, otherwise the run time would become too long. Increasing the $\mathrm{pH}$ above 5.4 results in shortening the run time together with a loss of resolution. The concentration of methanol should not vary by more than $1 \%$. Increasing the concentration of methanol above $4 \%$ results in shortening the run time with a concomitant loss of resolution. Decreasing the percentage of methanol below $2 \%$ results in a dramatic loss of efficiency and resolution. In the same way, the best efficiency and resolution were obtained at $30{ }^{\circ} \mathrm{C}$. 

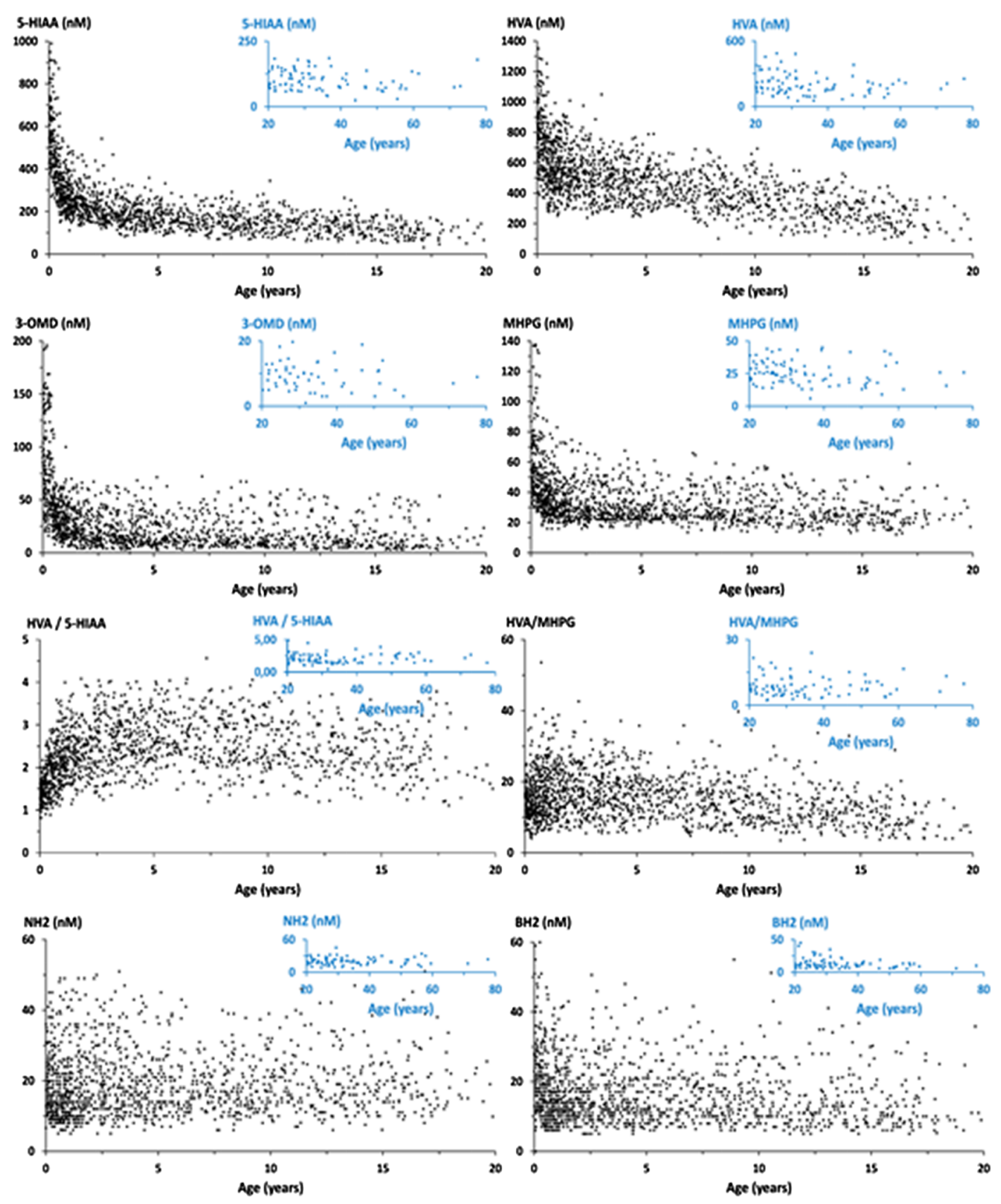

Figure 3. Variation of metabolite concentrations in human CSF with patient age.

We checked the absence of possible endogenous interferences including DOPA, dopamine, dihydroxy-phenylacetic acid, serotonin, and 3-methoxy-tyramine, which is the intermediate degradation product of dopamine leading to HVA. All of these metabolites are oxidizable compounds that could coelute with the targeted metabolites. Figure S2 shows that under our conditions, there is no coeluting peak with the used internal standard (IS). Dopamine elutes before the IS with a resolution higher than 1.3. Although serotonin and 3MT are not separated, these metabolites cannot interfere with 5-HIAA because their coeluting peaks are well-separated from the latter with a resolution higher than 1.4.

Proposed Method. Taken together, these analytical developments led us to adopt the following chromatographic conditions: separations were performed on an ACQUITY UPLC HSS T3 $(2.1 \times 100 \mathrm{~mm}$ and $1.8 \mu \mathrm{m})$ column protected by an
ACQUITY UPLC HSS T3 VanGuard precolumn $(2.1 \mathrm{~mm} \times 5$ $\mathrm{mm}$ and $1.8 \mu \mathrm{m}$ ), with a mobile phase consisting of $\mathrm{pH} 5.2$ and $0.05 \mathrm{M}$ sodium citrate/methanol $(97 / 3, \mathrm{v} / \mathrm{v})$ delivered at a flow rate of $0.5 \mathrm{~mL} / \mathrm{min}$ at $30{ }^{\circ} \mathrm{C}$. The ECD of the neurotransmitter metabolites is performed at $+600 \mathrm{mV}$ at the second electrode, and the sequential fluorescence detection of pterins was performed at $\lambda_{\mathrm{ex}} 350 \mathrm{~nm}$ and $\lambda_{\mathrm{em}} 450 \mathrm{~nm}$ after coulometric oxidation at the same potential. The final run time was $10 \mathrm{~min}$.

Method Evaluation. The identification of metabolites and pterins in authentic CSF samples was based on their retention factors and their electrochemical and fluorescence properties as well as proportional increases of corresponding peak areas after spiking with known amounts of each target compound (Table S2).

All unknown peaks are well-separated from the target metabolites under our chromatographic conditions. After more 
than 8 years of experience and more than 1500 handled CSF samples, we never observed any interfering peak at the level of the IS.

In some rare cases, probably because of the treatment, an interfering peak may coelute with 3-OMD. In these cases, this peak disappears just by decreasing the potential of the working electrode from 0.6 to $0.4 \mathrm{~V}$. It is then necessary to calibrate the method at $0.4 \mathrm{~V}$ to determine the 3-OMD concentration. Nevertheless, such an interfering peak cannot influence the diagnosis because the diagnosis is not only based on the variation of a metabolite alone but rather based on the modification of the CSF metabolic profile, as shown in Table 1.

Table S1 summarizes the linearity data and LOQs for target analytes. The method was linear for all compounds over the calibration range with a correlation coefficient $\left(R^{2}\right)$ higher than 0.99 for all instances. However, the intercepts are negative in all instances. Hence, with this regression model, it will not be possible to make a prediction for a point that is outside the range of the data.

Within-run and between-run precision for standard solutions did not exceed $9.7 \%$ for all instances (Table S2). Within-run precision for authentic CSF samples spiked with known amounts of metabolites and pterins did not exceed $9.5 \%$ with a recovery higher than $91.8 \%$ for all instances. For each compound, the recovery was determined after analyzing a pool of authentic CSFs before $\left(P_{0}\right)$ and after $\left(P_{s}\right)$ spiking it with a known amount of the corresponding analyte, by calculating the ratio: obtained amount $\left(P_{\mathrm{s}}\right)$ versus the theoretical amount $\left(\mathrm{Th}=P_{0}+\right.$ added concentration $)$ that gives $\left[R=\left(P_{s} / \mathrm{Th}\right) \times 100\right]$. Furthermore, there was no significant difference between within-run precision for spiked CSF samples and standard solutions as evidenced by $p$ values greater than 0.05 , with recoveries higher than $91.8 \%$ for all instances (Table S2), thus indicating the absence of significant matrix effect. Hence, we used aqueous standard samples rather than authentic pooled CSF ( $\mathrm{p}$-CSF) samples for method calibration.

We checked the stability of the samples at $10{ }^{\circ} \mathrm{C}$ in the dark in the injector after several hours. We did not observe significant differences for $24 \mathrm{~h}$. After $24 \mathrm{~h}, \mathrm{NH} 2$ concentrations decreased by about $15 \%$, whereas $\mathrm{BH} 2$ concentrations increased by about $10 \%$ of their respective initial values (Figure S3). Hence, using this method, the samples maintained at $10{ }^{\circ} \mathrm{C}$ in the dark should be analyzed within $24 \mathrm{~h}$ following defrosting.

In the same way, we checked the stability of the samples frozen at $-80{ }^{\circ} \mathrm{C}$ immediately after collection. Compared with the initial values determined at day 4 after collection (and extemporaneous defrosting), the data obtained for the aliquots of the same samples defrosted after 12 months of storage at -80 ${ }^{\circ} \mathrm{C}$ showed no significant differences. Hence, we avoided adding a preservative agent to the samples, thus facilitating the collection procedure.

Age-Related Reference Ranges of Neurotransmitter Metabolites and Pterins in Cerebrospinal Fluid. Figure 3 shows the distribution of the data obtained with the proposed method for 1516 CSF samples collected from 1386 infants and children aged 1 day to 16 years (median 3.34 years) and 130 patients aged $16.01-80$ years (median 21 years) without known neurotransmitter disorder or 5-MTHF deficiency.

The first PCA model was built with HVA, 5-HIAA, MHPG, 3OMD, 5-OTRP, BH2, and $\mathrm{NH} 2$ as variables and 1516 observations remaining after removing the outliers corresponding to the known 19 cases of neurotransmitter disorders and 115 cases of immune system activation. Figure 4A shows the score
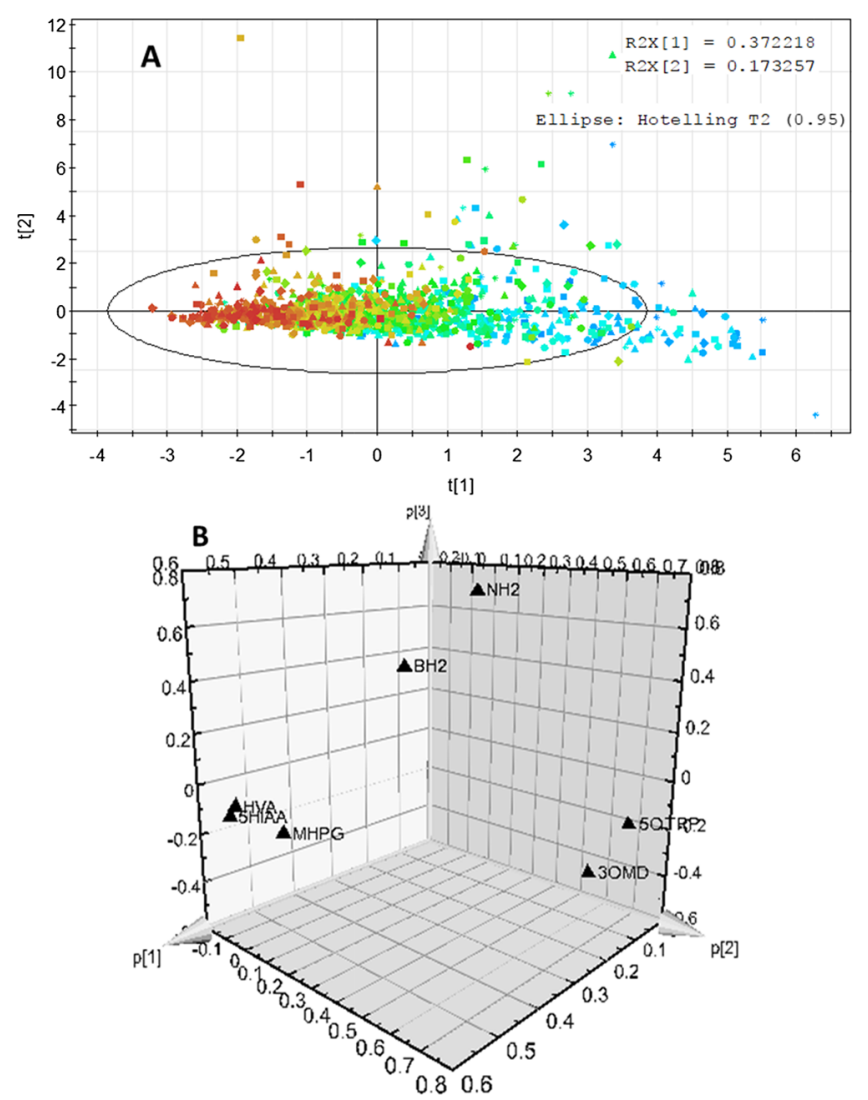

Figure 4. CP analysis with HVA, 5-HIAA, MHPG, 3-OMD, 5OH-Trp (5-HTrp), BH2, and $\mathrm{NH} 2$ as variables and 1516 observations. (A) Score plot and (B) loading plot.

plot where observations are colored according to the patient's age, from blue for the lowest values to red for the highest values. Principal components (CPs) 1 and 2 account for 37 and 17\% of the total variance, respectively. There is an evolution as a function of the age; however, the overlap of the colored points indicates that it will be difficult to distinguish age intervals.

In a second step, we considered only HVA, 5-HIAA, and MHPG, which are the main metabolites of dopamine, serotonin, and norepinephrine, respectively. The score plot still indicates that there is an obvious evolution with age, but it is still difficult to distinguish the age intervals (Figure S4A). Nevertheless, the loading plot (Figure S4B) clearly shows that HVA and 5-HIAA are the main contributors to $\mathrm{CP} 1$ and that they are strongly correlated. MHPG also contributes to CP 1 but to a less extent. MHPG mainly contributes to CP 2 (Figure S4B). This makes sense once we consider the metabolic pathway of these metabolites (Figure 1). Whereas HVA and 5-HIAA are derived from dopamine and serotonin, which are the products of the same enzyme, namely, AADC, MHPG is derived from norepinephrine, which depends on the action of an additional enzyme, namely, DHB (Figure 1). This is also true for 3-OMD and 5-HTrp, which are the substrates of AADC but the products of two different hydroxylases, $\mathrm{TH}$ and $\mathrm{TrpH}$ (Figure 1). The loading plot (Figure S4C) obtained in the third step clearly shows that the latter mainly contributes to $\mathrm{CP} 2$, which separates them.

In the fourth step, we added $\mathrm{BH} 2$, and we observed that this parameter is a major outlier, contributing to a third CP (Figure S4D). This also makes sense as $\mathrm{BH} 2$ results from the slow isomerization of $\mathrm{qBH} 2$, which is the oxidized intermediate of 
Table 2. Reference Ranges for CSF Concentrations of Neurotransmitter Metabolites and Pterins ${ }^{a}$

\begin{tabular}{|c|c|c|c|c|c|c|c|}
\hline \multirow[b]{2}{*}{ groups } & \multicolumn{7}{|c|}{ mean (med.) } \\
\hline & 5-HIAA & HVA & HVA/5-HIAA & 3-OMD & MHPG & $\mathrm{NH} 2$ & $\mathrm{BH} 2$ \\
\hline $\mathrm{A}(n=27)$ & $661(620)$ & $868(838)$ & $1.31(1.37)$ & $109(100)$ & $62(56)$ & $19(17)$ & $27(26)$ \\
\hline $0-1$ month & $426-1186$ & $405-1430$ & $0.81-1.73$ & $37-156$ & $37-110$ & $7-36$ & $9-59$ \\
\hline A vs B & $p=0.002$ & $p=0.111$ & $p=0.122$ & $p<0.001$ & $p=0.172$ & $p=0.543$ & $p=0.030$ \\
\hline $\mathrm{B}(n=166)$ & $468(441)$ & $726(700)$ & $2(2)$ & $74(61)$ & $54(47)$ & $18(15)$ & $21(18)$ \\
\hline 1 month to 0.5 year & $185-990$ & $274-1487$ & $0.8-3.7$ & $11-250$ & $18-138$ & $7-65$ & $6-69$ \\
\hline B vs $C$ & $p<0.001$ & $p<0.001$ & $p<0.001$ & $p<0.001$ & $p<0.001$ & $p=0.704$ & $p<0.001$ \\
\hline $\mathrm{C}(n=457)$ & $249(235)$ & $555(539)$ & $2.33(2.23)$ & $32(26)$ & $35(31)$ & $19(16)$ & $16(14)$ \\
\hline $0.5-3$ years & $111-631$ & $174-1176$ & $0.79-4.95$ & $4-209$ & $11-106$ & $5-50$ & $5-51$ \\
\hline $\mathrm{C}$ vs $\mathrm{D}$ & $p<0.001$ & $p<0.001$ & $p<0.001$ & $p<0.001$ & $p<0.001$ & $p=0.667$ & $p=0.758$ \\
\hline $\mathrm{D}(n=328)$ & $179(170)$ & $473(463)$ & $2.78(2.71)$ & $21(15)$ & $30(26)$ & $19(17)$ & $16(113)$ \\
\hline $3-7$ years & $46-583$ & $62-1185$ & $1.29-5.96$ & $3-179$ & $8-67$ & $6-69$ & $5-48$ \\
\hline $\mathrm{D}$ vs $\mathrm{E}$ & $p<0.001$ & $p<0.001$ & $p=0.159$ & $p=0.490$ & $p=0.490$ & $p=0.498$ & $p=0.666$ \\
\hline $\mathrm{E}(n=222)$ & $156(149)$ & $402(386)$ & $2.69(2.63)$ & $20(13)$ & $30(27)$ & $19(17)$ & $16(14)$ \\
\hline $7-11$ years & $57-406$ & $81-815$ & $1.20-4.61$ & $4-113$ & $14-66$ & $6-40$ & $6-55$ \\
\hline E vs $F$ & $p<0.001$ & $p<0.001$ & $p<0.001$ & $p=0.069$ & $p=0.003$ & $p=0.669$ & $p=0.009$ \\
\hline $\mathrm{F}(n=186)$ & $132(129)$ & $309(300)$ & $2.41(2.34)$ & $17(12)$ & $27(10)$ & $19(17)$ & $14(12)$ \\
\hline $11-16$ years & $46-276$ & $67-669$ & $0.85-5.74$ & $3-69$ & $12-71$ & $6-47$ & $5-41$ \\
\hline F vs $G$ & $p<0.001$ & $p<0.001$ & $p<0.001$ & $p=0.656$ & $p=0.913$ & $p=0.100$ & $p=0.421$ \\
\hline $\mathrm{G}(n=130)$ & $113(112)$ & $241(215)$ & $2.20(2.07)$ & $18(12)$ & $27(25)$ & $21(20)$ & $14(11)$ \\
\hline over 16 years & $40-186$ & $65-488$ & $1.04-4.90$ & $3-55$ & $12-59$ & $7-51$ & $5-45$ \\
\hline \multicolumn{8}{|c|}{ Known Metabolic Disorders $(n=18)$ and Elevated Levels of NH2 $(n=115)$} \\
\hline Segawa $(n=2) 4.2$ and 5.6 years & $10-17$ & $15-20$ & $1.50-1.18$ & $2-3$ & $4-6$ & $2-4$ & $1-3$ \\
\hline SR $(n=1) 27.88$ years & 21 & 56 & 0.72 & 25 & 3 & 35 & 67 \\
\hline $\mathrm{TH}(n=11) 0.01-18.63$ years & $212(189)$ & $129(117)$ & $0.62(0.62)$ & $40(22)$ & $35(33)$ & $15(12)$ & $28(26)$ \\
\hline \multirow{3}{*}{$\operatorname{AADC}^{b}(n=3) 0.46-2.40$ years } & $104-405$ & $44-256$ & $0.31-1.1$ & $5-167$ & $7-71$ & $8-49$ & $6-58$ \\
\hline & $9(3)$ & $15(15)$ & $5.55(7.50)$ & $1939(2500)$ & $28(25)$ & $15(17)$ & $21(21)$ \\
\hline & $2-23$ & $3-27$ & $0.15-9$ & $252-3065$ & $7-50$ & $10-55$ & $14-47$ \\
\hline PTPS $(n=1) 24.46$ years & 15 & 109 & 7.38 & 29 & 11 & 4 & 5 \\
\hline DTDS $(n=1) 0.92$ year & 302 & 2426 & 8.03 & 96 & 38 & 48 & 7 \\
\hline elevated levels of NH2 $(n=115)$ & $258(232)$ & $498(490)$ & $2.09(2.11)$ & $44(29)$ & $34(28)$ & $316(184)$ & $38(24)$ \\
\hline $0.02-60.64$ years & $26-732$ & $23-1091$ & $0.49-3.84$ & $3-116$ & $4-76$ & $90-2500$ & $21-91$ \\
\hline
\end{tabular}

${ }^{a}$ Results are expressed in nanometers as average (median) and range. ${ }^{b}$ For AADC, the main concentration of 5-HTrp is $505 \mathrm{nM}$, with a median of $316 \mathrm{nM}$ (range: 300-900 $\mathrm{nM}$ ).

BH4, the common cofactor of TrpH and TH (Figure 1). Finally, in the last step, we added $\mathrm{NH} 2$, and we observed that it also contributes to the third component while it is slightly correlated with $\mathrm{BH} 2$. The latter is being separated by the axis of the third component (Figure 4B). This also makes sense once we consider that $\mathrm{NH} 2$ is the precursor of $\mathrm{BH} 4$ (Figure 1).

In conclusion, although the PCA model well-establishes the evolution of the main metabolites with the age of patients as well as the correlations between these parameters, it remains difficult to distinguish with this model the relevant age intervals and thus to establish the reference ranges.

Hence, we investigated the normality of the data by using both graphical representation (histograms and $\mathrm{Q}-\mathrm{Q}$ plots) and Shapiro-Wilk or Shapiro-Francia tests depending on the kurtosis. ${ }^{21}$ In most cases, the data were not normally distributed, but they are log-normally distributed as shown by $p$-values less than 0.001 (Table S3). Nevertheless, we used Pearson correlation coefficient to study the correlation between metabolite concentrations and age.

As expected, ${ }^{16,18,22,23}$ a negative correlation was observed between most metabolites and age in the whole group of patients (HIAA: $r=-0.5549, p<0.0001$; HVA: $r=-0.5602, p<0.0001$; 3-OMD: $r=-0.3929, p<0.0001$; MHPG: $r=-0.3355, p<$ 0.0001 ; and BH2: $r=-0.1459, p<0.0001)$. Whereas the HVA/ MHPG ratio showed a slight negative correlation with age $(r=$
-0.1829 and $p<0.0001)$, HVA/HIAA and HVA/3-OMD ratios showed a positive correlation with age $(r=0.2365, p<0.0001$ and $r=0.0790, p=0.0157$, respectively). By contrast, $\mathrm{NH} 2 \mathrm{did}$ not exhibit any correlation with age $(r=0.0373$ and $p=0.2613)$.

In a second step, we grouped the patients by age month by month for the first year and year by year for the patients older than one year. We also grouped the patients according to the previously published data. ${ }^{16}$ Considering a $p$-value less than 0.05 statistically significant, data were mostly log-normally distributed in all groups older than one month (Table S3).

Age groups were then compared by using Student's $t$-test. For HVA and 5-HIAA concentrations, the differences between the groups older than 1 month were significant. However, in contrast to previous studies, ${ }^{16} \mathrm{HVA}, \mathrm{MHPG}$, and $\mathrm{BH} 2$ concentrations in the group of newborns younger than one month were not statistically different from the group aged from 1 to 6 months. This discrepancy may be attributed to the lower number of patients included in previous studies. ${ }^{16} 5$-HTrp was found to be less than $12 \mathrm{nmol} / \mathrm{L}$ in every CSF sample (Table 2). Nevertheless, the correlation with age among the target metabolites was mostly similar to those previously published. ${ }^{16,22}$

Irrespective of the age or sex, strong positive correlations were observed between HVA and HIAA $(r=0.7742$ and $p<0.0001)$, HVA and MHPG $(r=0.4446$ and $p<0.0001)$, HVA and 3-OMD $(r=0.4483$ and $p<0.0001)$, MHPG and 3-OMD $(r=0.5918$ and 

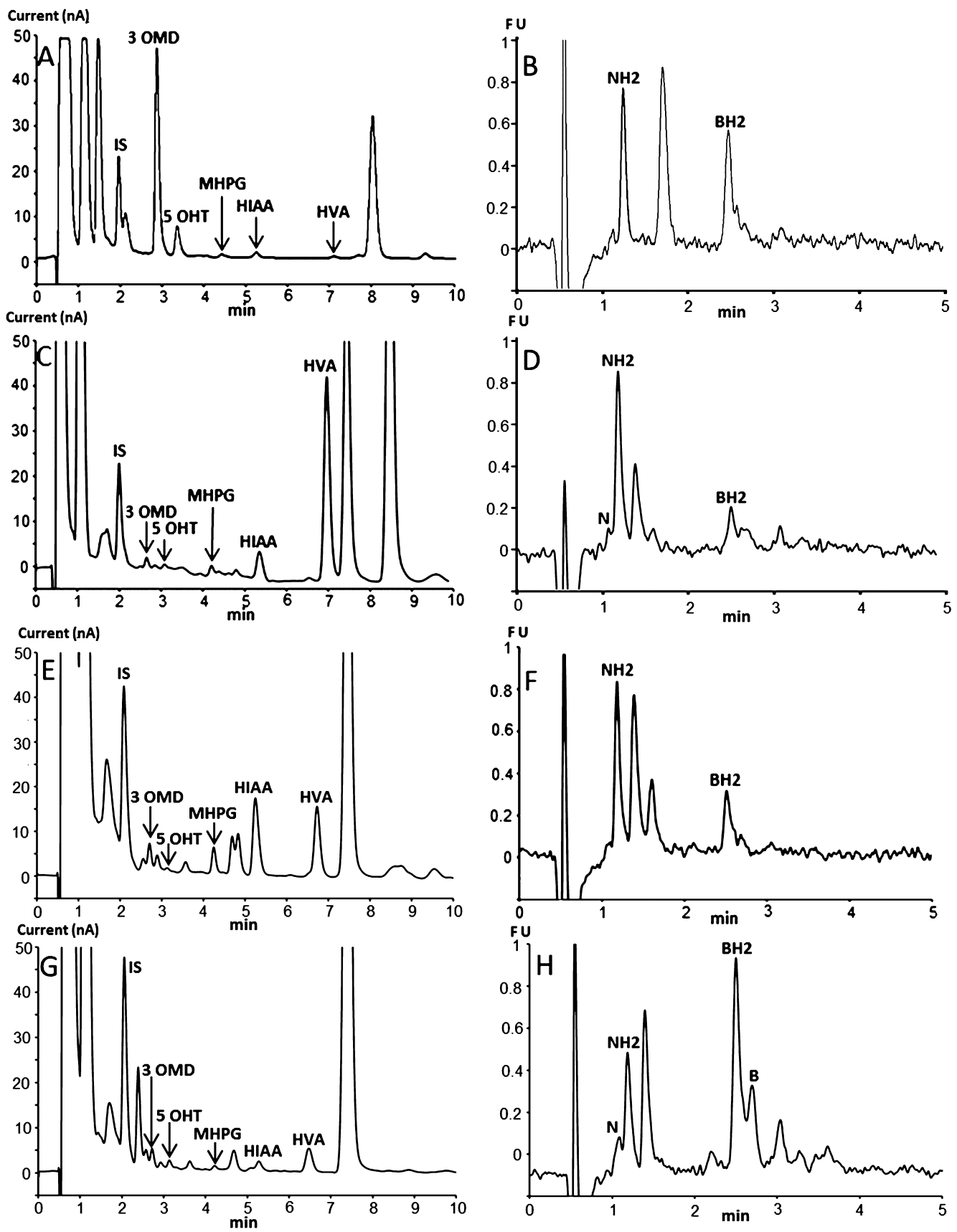

Figure 5. Chromatographic profiles obtained with the proposed method for some patients with known neurotransmitter disorders. (A,B) AADC deficiency, (C,D) DTDS, (E,F) TH deficiency, and (G,H) SR deficiency. The CSF sample (C) has been diluted twice before injection. The injection volume was $25 \mu \mathrm{L}$ for $(\mathrm{A}, \mathrm{C})$ and $50 \mu \mathrm{L}$ for $(\mathrm{E}, \mathrm{G})$.

$p<0.0001)$, HVA and BH2 $(r=0.2781$ and $p<0.0001)$, and HIAA and $\mathrm{BH} 2(r=0.2339$ and $p<0.0001)$. $\mathrm{BH} 2$ and $\mathrm{NH} 2$ also exhibited a slightly positive correlation $(r=0.1241$ and $p=$ 0.0002). The correlations between HIAA, HVA, and $\mathrm{BH} 2$ confirm that the $\mathrm{BH} 2$ concentration well-reflects the $\mathrm{BH} 4$ availability in the CSF.

Table 2 summarizes the reference intervals obtained with the proposed method. Except for the patients aged less than one month, which are not different from those aged between 1 and 6 months, the obtained reference ranges are similar to those previously obtained in other countries by classical methods. ${ }^{16,22,23}$ These results thus strengthen the validation of the proposed method.
Analysis of the Known Cases of Dopamine and Serotonin Metabolism Disorders. The proposed method was applied to the diagnosis of several known inborn errors of dopamine and serotonin metabolism including 2 cases of Segawa syndrome, 1 case of sepiapterin reductase deficiency, 3 cases of AADC deficiency, 1 case of dopamine transporter deficiency, 1 case of PTPS deficiency, 11 cases of tyrosine hydroxylase deficiency, as well as more than 100 cases of intracerebral immune activation with elevated levels of NH2 (Table 2, Figure 5). The obtained results (Table 2) confirmed the expected CSF pattern of changes in pterins and monoamine metabolites in patients.

Otherwise, the proposed method allows the unambiguous detection of $\mathrm{NH} 2$ increase, which is a known biomarker of 

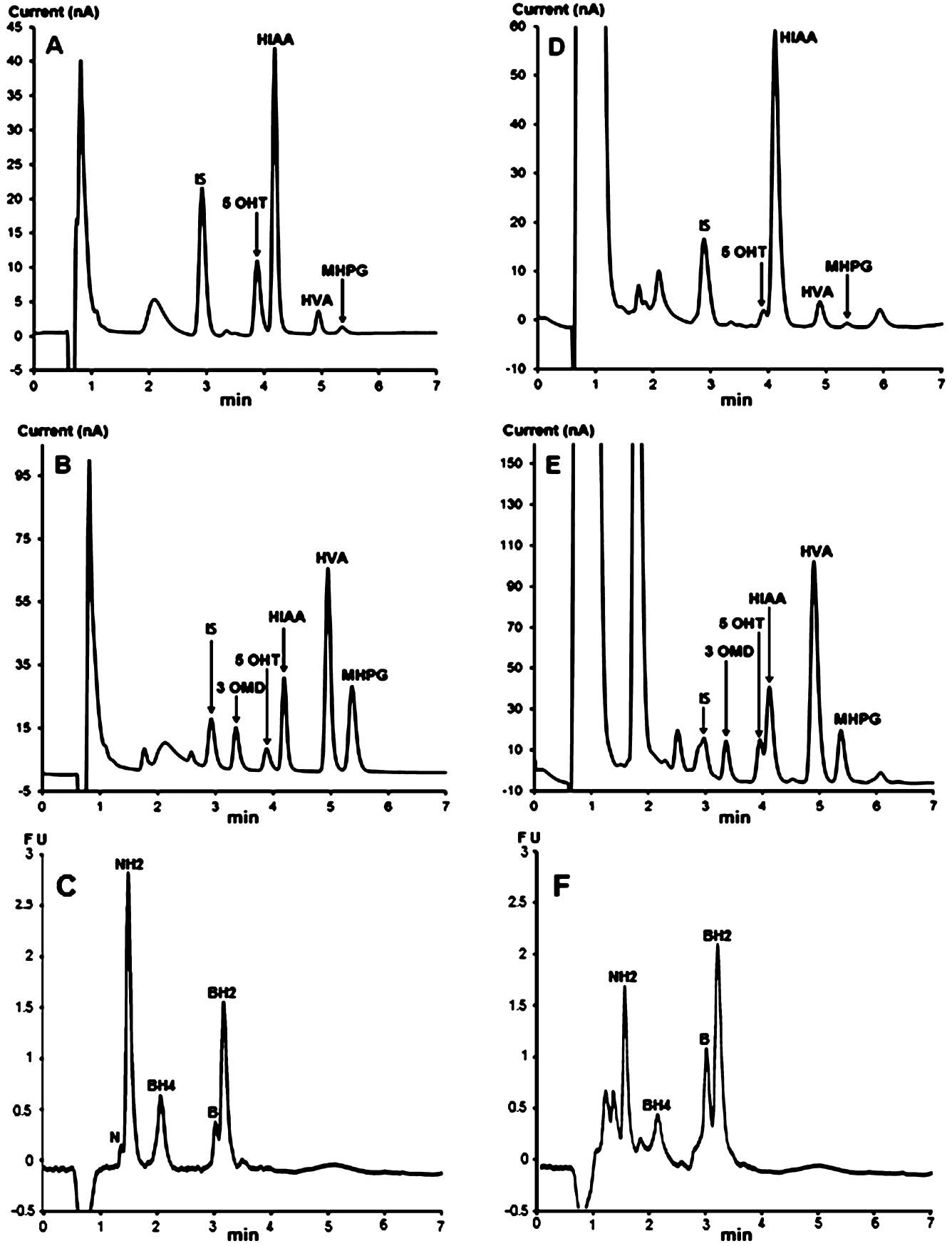

Figure 6. Chromatographic profiles of a standard mixture and a CSF sample obtained with a pH 7.4 mobile phase. (A-C) Standard mixture with ECD at (A) $+400 \mathrm{mV}$ for the first electrode, (B) $+600 \mathrm{mV}$ for the second electrode, followed by (C) sequential fluorescence detection. (D-F) CSF sample with $\mathrm{ECD}$ at $(\mathrm{D})+400 \mathrm{mV}$ for the first electrode, $(\mathrm{E})+600 \mathrm{mV}$ for the second electrode, followed by (F) sequential fluorescence detection (see Figure 2 for the other chromatographic conditions).

immune system activation. ${ }^{24}$ It is worth noting that about $7 \%$ of the analyzed CSF samples exhibited elevated levels of $\mathrm{NH} 2$. Hence, the proposed method is a useful tool for the diagnosis of not only neurotransmitter disorders but also all cases of intracerebral immune system activation, which appear much more frequent than the former in the studied population.

Simultaneous Determination of $\mathrm{BH} 4$. Although $\mathrm{BH} 4$ determination is not mandatory for the etiologic diagnosis of neurotransmitter disorders, the determination of this cofactor may be interesting for metabolism and pharmacokinetics studies. $^{18}$
As $\mathrm{BH} 4$ cannot be detected at $\mathrm{pH} 5.2$ and can be detected at $\mathrm{pH} \mathrm{7.4,}{ }^{18}$ we later developed a variant of the proposed method able to simultaneously determine $\mathrm{BH} 4$. In fact, this variant only differs in the $\mathrm{pH}$ of the mobile phase, that is, $\mathrm{pH} 7.4$ instead of $\mathrm{pH}$ 5.2 as used in the proposed method.

Setting the $\mathrm{pH}$ of the mobile phase at 7.4 resulted in the simultaneous detection of $\mathrm{BH} 4$ in addition to all target compounds (Figure 6) with a good resolution but with different selectivities as reflected in the changes of the elution order. Under these conditions, the optimum sensitivity is obtained at an oxidation potential of $+400 \mathrm{mV}$ (first working electrode) for 5HTrp and 5-HIAA (Figure 6A) and an oxidation potential of 
$+600 \mathrm{mV}$ (second working electrode) for 3-OMD, HVA, and MHPG (Figure 6B). The signal decrease for 5-HTrp and 5HIAA at $+600 \mathrm{mV}$ is linked to the consumption of a large part of these metabolites at the first electrode set at $+400 \mathrm{mV}$. To optimize the transformation of $\mathrm{BH} 4$ into its fluorescent counterpart, it is mandatory to use both electrodes for postcolumn oxidation of this pterin.

Method evaluation showed that the analytical performances of this variant method operating at $\mathrm{pH} 7.4$ are similar to those of the proposed method operating at $\mathrm{pH} 5.2$ (Table S4). Also, the results obtained with the variant operating at $\mathrm{pH} 7.4$ for real-CSF samples $(n=50)$ showed a good correlation with those obtained with the proposed method operating at $\mathrm{pH} 5.2$ with a correlation coefficient higher than 0.99 for all target metabolites and pterins (Figure S5).

As the stability of the CSF samples at $\mathrm{pH} 7.4$ is limited to $6 \mathrm{~h}$ in an autosampler at $10{ }^{\circ} \mathrm{C}^{18}$ instead of $24 \mathrm{~h}$ for the proposed method and because the stationary phase is less stable at $\mathrm{pH} 7.4$ than under the conditions of the proposed method operating at $\mathrm{pH}$ 5.2, we recommend using the proposed method for the routine diagnosis of neurotransmitter disorders. Nevertheless, the derived method operating at $\mathrm{pH} 7.4$ can be used for the simultaneous quantification of $\mathrm{BH} 4$ if needed as well as for checking the results of any CSF sample suspected to contain some potential interferences. In such cases, the difference in selectivity observed at $\mathrm{pH} 7.4$ may help to detect the possible unexpected exogenous interfering compounds coeluting with the targeted compounds at $\mathrm{pH} 5.2$ and vice versa.

After more than 6 years of experience, the only possible interfering peaks we observed are those present on the chromatograms of Figures 2, 5, and 6. As shown in these figures, all unknown peaks are well-separated from those of the targeted metabolites.

In fact, the only possible interferences that may occur are only unexpected exogenous interferences linked to unknown treatments. If there are some interferences coeluting with analytes and resulting in one peak, which is obviously possible for any method of separation of complex mixtures, it would be easy to suspect their presence. Indeed, as the diagnosis of neurotransmitter disorders is based on the characteristic CSF patterns (Table 1), such an interference would result in an unusual doubtful metabolic profile. Hence, an alternative method of separation with a different selectivity may be helpful to highlight such an interference.

\section{CONCLUSIONS}

An UHPLC method for a one-step rapid diagnosis of inborn errors of metabolism of dopamine and serotonin is presented. The use of an embedded-polar-group-bonded phase (ACQUITY HSS T3) and sequential coulometric and fluorescence detections allows the simultaneous quantification of neurotransmitter metabolites and pterins of interest. All target neurotransmitter metabolites and pterins were quantified in a small volume of CSF $(50 \mu \mathrm{L})$ using a single filtration step for sample preparation and analysis.

As the samples are stable without adding any antioxidant agent for $24 \mathrm{~h}$ in an autosampler at $10^{\circ} \mathrm{C}$ and as the run time is $10 \mathrm{~min}$, the number of samples including calibrators and quality control that can be analyzed per run is 144 .

The application of the proposed method to the analysis of 1516 human CSF samples without known neurotransmitter disorders allowed us to give the age-related reference ranges for key metabolites and pterins among French population.
Furthermore, the application of the proposed method to several cases of known enzymatic defects confirmed the expected CSF pattern of changes in pterins and monoamine metabolites in patients.

Although previous methods using MS/MS detection were less sensitive than FD for pterin quantification, ${ }^{18}$ recent advances in MS/SM detection ${ }^{19}$ notably in terms of sensitivity show that the latter mode of detection is now able to replace the former. As MS/MS detection is less dependent on the resolution of the separation, the translation of the proposed method to UHPLCMS/MS is one avenue to improve the overall throughput of this method. For the time being, the proposed method is quite appropriate for the laboratories not already equipped with the LC-MS/MS technology.

\section{METHODS}

Chemicals and Reagents. All reagents were purchased from Sigma (Saint-Quentin Fallavier, France) and were used without further purification.

Patient Samples. The CSF samples were collected from 2008 to 2014 by lumbar puncture as previously described. ${ }^{18}$ Lumbar punctures were performed in several French hospitals covering the entire French territory as part of normal clinical management and research activities aiming to enhance the diagnosis of neurological disorders of unknown origin with the written informed consent of parents or legal representatives of each patient. This study was performed according to French public health regulations (Code de la santé publique-Article L1121-3, modified by Law no. 2011-2012, December 29 2011Article 5). Samples were collected in five fractions of $0.3 \mathrm{~mL}$ each as previously described ${ }^{18}$ and were immediately frozen with liquid nitrogen and then stored at $-80{ }^{\circ} \mathrm{C}$ until analysis. The exclusion criteria were traumatic punctures, inadequate collection and preservation of the samples, and L-DOPA and $\mathrm{BH} 4$ treatments. Determination of 5-methyl tetrahydrofolate by HPLC-FD ${ }^{17}$ was performed for all patients.

The collected CSF samples were divided into three groups. Group 1 includes CSF samples collected by lumbar puncture from 1386 infants and children aged 1 day to 16 years (median 3.34 years) and 130 patients aged 16.01 to 80 years (median 21 years). Group 1 was considered as a reference population with the same inclusion criteria as previously described (ref 18). This group includes patients suffering from several neurological disorders with initially unknown etiology including movement disorders with or without encephalopathy, epileptic or neurodegenerative encephalopathy, and meningoencephalitis. Group 2 includes CSF samples with known neurotransmitter disorders collected from 16 infants and children (aged 1 day to 6.63 years, median 2.36 years) and 3 adults (aged 18.63 to 27.88 years, median 24.46 years). Group 3 includes CSF samples collected from 115 patients (aged 0.02 to 64.64 years, median 25.6 years) with known immune system activation.

Chromatographic Conditions and Sample Preparation. Preliminary HPLC separations were performed on a Dionex Summit HPLC system (Les Ulis, France). For the development of the method, the HPLC system was coupled to a model 5011 cell controlled by a Coulochem 5100A module (ESA, France) connected to a JASCO FP 920 detector equipped with a $16 \mu \mathrm{L}$ standard cell. For simultaneous separation of neurotransmitter metabolites and pterins, we used an Atlantis T3 $(4.6 \times 150 \mathrm{~mm}$ and $3 \mu \mathrm{m})$ column (Waters, France). The mobile phase consisted of a mixture of $\mathrm{pH} 5.2$ and $0.05 \mathrm{M}$ citrate buffer and methanol $(97 / 3, \mathrm{v} / \mathrm{v})$. The flow rate was set at $0.5 \mathrm{~mL} / \mathrm{min}$ at 
$30{ }^{\circ} \mathrm{C}$. The metabolites present in the effluent were measured at the second electrode with a potential set at $400 \mathrm{mV}$, whereas the fluorescence of pterins was sequentially measured at $450 \mathrm{~nm}$ after column electrooxidation at the same potential and excitation at $350 \mathrm{~nm}$

For the UHPLC separations, we used a Waters ACQUITY UPLC system connected to an ESA Coulochem III electrochemical detector equipped with a 6011 model cell followed by an ACQUITY UPLC fluorescence detector. For the separation of neurotransmitter metabolites and pterins, we used an ACQUITY UPLC HSS T3 $(2.1 \times 100 \mathrm{~mm}$ and $1.8 \mu \mathrm{m})$ column (Waters, France). The mobile phase consisted of a mixture of $\mathrm{pH}$ 5.2 and $0.05 \mathrm{M}$ citrate buffer and methanol $(97 / 3, \mathrm{v} / \mathrm{v})$. The flow rate was set at $0.5 \mathrm{~mL} / \mathrm{min}$ at $30^{\circ} \mathrm{C}$. The metabolites and pterins present in the effluent were sequentially measured under the same conditions as for the HPLC separation but with a potential set at $600 \mathrm{mV}$. We changed the potential to $600 \mathrm{mV}$ to reach the limiting current. Obviously, the potential shift is due to the used reference electrodes that are likely different between the two used Coulochem models.

For sample preparation, $50 \mu \mathrm{L}$ of CSF or calibrator or QC sample was diluted $(1 / 1, \mathrm{v} / \mathrm{v})$ in a solution of 2,5 dihydroxybenzoic acid $(250 \mathrm{nM})$ used as an IS prepared in the mobile phase, before filtration in a 5000 MWCO PES Vivaspin 500 filter (Sartorius, Aubagne, France) and centrifugation (10 min at $12000 \mathrm{~g}$ and $\left.4{ }^{\circ} \mathrm{C}\right)$. The resulting filtrate $(50 \mu \mathrm{L})$ was injected into the chromatograph. However, to respect the linearity of the method, the injected volume must be appropriately reduced for some CSF samples containing high levels of metabolites. Also, to avoid the dramatic reduction in the area of the IS peak, some samples containing high levels of metabolites must be appropriately diluted before addition of the IS and injection.

Calibrators and Quality Controls. Stock standard solutions were prepared by dissolving $400 \mu \mathrm{M}$ of biopterin (B), neopterin $(\mathrm{N}), \mathrm{BH} 2$, and $\mathrm{NH} 2$ in $0.1 \mathrm{M} \mathrm{HCl}$ or $1 \mathrm{mM}$ of HVA, HIAA, 3-OMD, 5-HTrp, and MHPG in deionized water. After rapid preparation, aliquots of the standard solutions were immediately frozen at $-80{ }^{\circ} \mathrm{C}$ until use.

To plot the calibration curves, the stock standard solutions of $\mathrm{N}, \mathrm{NH} 2, \mathrm{~B}$, and $\mathrm{BH} 2$ were diluted to give final concentrations of $5,12.5,25,50$, and $100 \mathrm{nM} / \mathrm{L}$ of each pterin. HVA was diluted to give final concentrations of $15,75,187.5,375,750$, and 1500 $\mathrm{nM} / \mathrm{L}$. HIAA was diluted to give final concentrations of 10,50 , 125, 250, 500, and $1000 \mathrm{nM} / \mathrm{L}$. MHPG, 3-OMD, and 5-HTrp were diluted to give final concentrations of $5,25,62.5,125,250$, and $500 \mathrm{nM}$.

Analytical recovery and precision studies were performed on the p-CSF samples spiked with 3-OMD, 5-HTrp, MHPG, HIAA, $\mathrm{HVA}, \mathrm{BH} 2, \mathrm{~B}$, and $\mathrm{NH} 2$ at three concentration levels (low, medium, and high). Aliquots of $200 \mu \mathrm{L}$ of p-CSF sample residues $(n=10)$ were spiked with $5,12.5$, and $50 \mathrm{nM} / \mathrm{L}$ of each pterin; $75,187.5$, and $750 \mathrm{nM} / \mathrm{L}$ of HVA; 50,125 , and $500 \mathrm{nM} / \mathrm{L}$ of HIAA, and 25, 62.5, and $250 \mathrm{nM} / \mathrm{L}$ of MHPG, 3-OMD, and 5HTrp. The basal content of the p-CSF sample was determined with the standard addition method (Skoog, DA. 1996). As internal quality control, we used aliquots of p-CSF frozen at -80 ${ }^{\circ} \mathrm{C}$ until analysis. As external QC (EQC), we used aliquots of diluted water $(1 / 100, v / v)$ "Special assays in urine" from ERNDIM (manufactured by the MCA Laboratory of the Queen Beatrix Hospital, Netherlands) stored at $-80{ }^{\circ} \mathrm{C}$ until analysis. This EQC is used to control the 5-HIAA and HVA levels. For the control of 3-OMD, 5-HTrp, and MHPG, we used their response factors as compared to those of 5-HIAA and HVA.

Statistics. The Shapiro-Wilk or Shapiro-Francia tests were used to check the normality of the data distribution. Histogram and normal probability plots were also drawn to graphically assess the normality of the data. Comparisons were performed with a two-tailed Student's $t$-test, after checking the homogeneity of variances using Fisher's test. The $p$ values less than 0.05 were considered statistically significant. Relationships between variables were examined using Pearson coefficient, linear regression, and analysis of variance. Statistical analysis was performed using MATLAB and excel.

\section{ASSOCIATED CONTENT}

S Supporting Information

The Supporting Information is available free of charge on the ACS Publications website at DOI: 10.1021/acsomega.7b01008.

Linearity, analytical recovery, and accuracy of the proposed method; Shapiro-Wilk normality test on population and log-transformed population; chromatographic profiles of a standard mixture and a CSF sample with the proposed method under HPLC conditions; and correlation between the results obtained with the proposed method at $\mathrm{pH} 5.2$ and at $\mathrm{pH} 7.4$ (PDF)

\section{AUTHOR INFORMATION}

\section{Corresponding Author}

*E-mail: fathi.moussa@u-psud.fr (F.M.).

\section{Author Contributions}

A.L., P.G., J.-Y.H., and F.M. performed research and analyzed the data. D.D., E.R., D.R., and T.B.D.V. managed the children and collected the CSF samples. R.C. provided material support. F.M. designed research and analyzed the data. A.L., P.G., and F.M. wrote the paper. All authors discussed the results and comments on the manuscript.

\section{Notes}

The authors declare no competing financial interest.

\section{ABBREVIATIONS}

3-OMD, 3-ortho-methyl DOPA; 5-HIAA, hydroxyl indole acetic acid; 5-HTrp, 5-hydroxytryptophan; AADC, amino acid decarboxylase; $\mathrm{AD}$, autosomal dominant; $\mathrm{AR}$, autosomal recessive; Bbiopterin $\mathrm{BH} 2$, biopterin $\mathrm{BH} 2$ dihydrobiopterin; $\mathrm{BH} 4$, tetrahydrobiopterin;; CSF, cerebrospinal fluid; DHPR, dihydropteridine reductase; DTDS, dopamine transporter deficiency syndrome; EQC, external quality control; FD, fluorescence detection; GTP-CH, guanosine triphosphate cyclohydrolase; HPLC, high-performance liquid chromatography; HVA, homovanillic acid; IQC, internal quality control; IS, internal standard; LOD, limit of detection; LOQ limit of quantification; MHPG, 3-methoxy-4-hydroxyphenylglycol; N, neopterin; NH2, dihydroneopterin; Phe, phenylalanine; PCD, pterin- $4 \alpha$-carbinolamine dehydratase; PTPS, 6-pyruvoyl tetrahydropterin synthase; $\mathrm{SR}$, sepiapterin reductase; $\mathrm{TH}$, tyrosine hydroxylase; UHPLC, ultrahigh-performance liquid chromatography

\section{REFERENCES}

(1) Hyland, K. Inherited disorders affecting dopamine and serotonin: critical neurotransmitters derived from aromatic amino acids. J. Nutr. 2007, 137, 1568S-1575S. 
(2) Longo, N. Disorders of biopterin metabolism. J. Inherited Metab. Dis. 2009, 32, 333-342.

(3) Kurian, M. A.; Gissen, P.; Smith, M.; Heales, S. J. R.; Clayton, P. T. The monoamine neurotransmitter disorders: an expanding range of neurological syndromes. Lancet Neurol. 2011, 10, 721-733.

(4) Koshimura, K.; Murakami, Y.; Tanaka, J.; Kato, Y. The role of 6Rtetrahydrobiopterin in the nervous system. Prog. Neurobiol. 2000, 61, 415-438.

(5) Blau, N.; Bonafé, L.; Blaskovics, M. E. Disorders of phenylalanine and tetrahydrobiopterin metabolism. In Physician's Guide to the Laboratory Diagnosis of Metabolic Diseases, 2nd ed.; Blau, N., Duran, M., Blaskovics, M. E., Gibson, K. M., Eds.; Springer: New York, 2003; pp 89-106.

(6) Roze, E.; Blau, N. Biogenic monoamine disorders. In Inherited Metabolic Disease in Adults: A Clinical Guide, 1st ed.; Hollak, C. E. M., Lachmann, R., Eds.; OUP: Oxford, USA, 2016; pp 203-208.

(7) Hyland, K. Clinical utility of monoamine neurotransmitter metabolite analysis in cerebrospinal fluid. Clin. Chem. 2008, 54, 633641.

(8) Allen, G. F. G.; Land, J. M.; Heales, S. J. R. A new perspective on the treatment of aromatic L-amino acid decarboxylase deficiency. Mol. Genet. Metab. 2009, 97, 6-14.

(9) Leu-Semenescu, S.; Arnulf, I.; Decaix, C.; Moussa, F.; Clot, F.; Boniol, C.; Touitou, Y.; Levy, R.; Vidailhet, M.; Roze, E. Sleep and Rhythm Consequences of a Genetically Induced Loss of Serotonin. Sleep 2010, 33, 307-314.

(10) Fukushima, T.; Nixon, J. C. Analysis of reduced forms of biopterin in biological tissues and fluids. Anal. Biochem. 1980, 102, 176-188.

(11) Howells, D. W.; Smith, I.; Hyland, K. Estimation of tetrahydrobiopterin and other pterins in cerebrospinal fluid using reversed-phase high-performance liquid chromatography with electrochemical and fluorescence detection. J. Chromatogr. B: Biomed. Sci. Appl. 1986, 381, 285-294.

(12) Koyama, E.; Minegishi, A.; Ishizaki, T. Simultaneous determination of four monoamine metabolites and serotonin in cerebrospinal fluid by "High-Performance" Liquid Chromatography with electrochemical detection; application for patients with Alzheimer's Disease. Clin. Chem. 1988, 34, 680-684.

(13) Tani, Y.; Ohno, T. Analysis of 6R- and 6S-tetrahydrobiopterin and other pterins by reversed-phase ion-pair liquid chromatography with fluorimetric detection by post-column sodium nitrite oxidation. $J$. Chromatogr. B: Biomed. Sci. Appl. 1993, 617, 249-255.

(14) Hubbard, K. E.; Wells, A.; Owens, T. S.; Tagen, M.; Fraga, C. H.; Stewart, C. F. Determination of dopamine, serotonin, and their metabolites in pediatric cerebrospinal fluid by isocratic high performance liquid chromatography coupled with electrochemical detection. Biomed. Chromatogr. 2010, 24, 626-631.

(15) Manini, P.; Andreoli, R.; Cavazzini, S.; Bergamaschi, E.; Mutti, A.; Niessen, W. M. A. Liquid chromatography-electrospray tandem mass spectrometry of acidic monoamine metabolites. J. Chromatogr. B: Biomed. Sci. Appl. 2000, 744, 423-431.

(16) Ormazabal, A.; García-Cazorla, A.; Fernández, Y.; FernándezÁlvarez, E.; Campistol, J.; Artuch, R. HPLC with electrochemical and fluorescence detection procedures for the diagnosis of inborn errors of biogenic amines and pterins. J. Neurosci. Methods 2005, 142, 153-158.

(17) Verbeek, M. M.; Blom, A. M.; Wevers, R. A.; Lagerwerf, A. J.; van de Geer, J.; Willemsen, M. A. A. P. Technical and biochemical factors affecting cerebrospinal fluid 5-MTHF, biopterin and neopterin concentrations. Mol. Genet. Metab. 2008, 95, 127-132.

(18) Guibal, P.; Lévêque, N.; Doummar, D.; Giraud, N.; Roze, E.; Rodriguez, D.; Couderc, R.; de Villemeur, T. B.; Moussa, F. Simultaneous Determination of All Forms of Biopterin and Neopterin in Cerebrospinal Fluid. ACS Chem. Neurosci. 2014, 5, 533-541.

(19) Arning, E.; Bottiglieri, T. LC-MS/MS analysis of cerebrospinal fluid metabolites in the pterin biosynthetic pathway. JIMD Reports; Springer, 2014; Vol. 29, pp 1-9.

(20) Bourcier, S.; Benoist, J.-F.; Clerc, F.; Rigal, O.; Taghi, M.; Hoppilliard, Y. Detection of 28 neurotransmitters and related compounds in biological fluids by liquid chromatography/tandem mass spectrometry. Rapid Commun. Mass Spectrom. 2006, 20, 14051421.

(21) Razali, N. M.; Wah, Y. B. Power comparisons of Shapiro-Wilk, Kolmogorov-Smirnov, Lilliefors and Anderson-Darling tests. J. Stat. Model. Anal. 2011, 2, 21-33.

(22) Hyland, K.; Surtees, R. A. H.; Heales, S. J. R.; Bowron, A.; Howells, D. W.; Smith, I. Cerebrospinal fluid concentrations of pterins and metabolites of serotonin and dopamine in a pediatric reference population. Pediatr. Res. 1993, 34, 10-14.

(23) Molero-Luis, M.; Serrano, M.; Ormazábal, A.; Pérez-Dueñas, B.; García-Cazorla, À.; Pons, R.; Artuch, R.; The Neurotransmitter Working Group. Homovanillic acid in cerebrospinal fluid of 1388 children with neurological disorders. Dev. Med. Child. Neurol. 2013, 55, 559-566.

(24) Hagberg, L.; Cinque, P.; Gisslen, M.; Brew, B. J.; Spudich, S.; Bestetti, A.; Price, R. W.; Fuchs, D. Cerebrospinal fluid neopterin: an informative biomarker of central nervous system immune activation in HIV-1 infection. AIDS Res. Ther. 2010, 7, 15. 